\title{
Does Dairy Food Intake Predict Arterial Stiffness and Blood Pressure in Men? Evidence from the Caerphilly Prospective Study
}

\author{
K. M. Livingstone ${ }^{1}$, J. A. Lovegrove ${ }^{1}$, J. R. Cockcroft $^{2}$, P. C. Elwood ${ }^{3}$, J. E. Pickering ${ }^{3}$ \\ and D. I Givens ${ }^{1}$ \\ ${ }^{1}$ Faculty of Life Sciences, University of Reading, Reading, ${ }^{2}$ Wales Heart Research Institute, Cardiff University, Cardiff \\ and ${ }^{3}$ School of Medicine, Cardiff University, Cardiff, UK
}

Arterial stiffness is an independent predictor of cardiovascular disease events and mortality ${ }^{(1)}$, and like blood pressure ${ }^{(2)}$, may be influenced by dairy food intake ${ }^{(3)}$. Few studies have investigated the effects of consumption of these foods on prospective measures of arterial stiffness. The present analysis aimed to investigate the prospective relationship between milk, cheese, cream and butter consumption with aortic pulse wave velocity, augmentation index, systolic (SBP) and diastolic blood pressure (DBP) and pulse pressure as well as crosssectional relationships between these foods and SBP, DBP, pulse pressure and metabolic markers using data from the Caerphilly Prospective Study. Included in this cohort were 2,512 men, aged 45-59 years, who were followed up at 5-year intervals for a mean of 22.8 years ( $n$ follow-up 787).

\begin{tabular}{|c|c|c|c|c|c|c|c|c|}
\hline & \multicolumn{4}{|c|}{ Cross sectional $^{1}$} & \multicolumn{4}{|c|}{ Longitudinal $^{2}$} \\
\hline & \multicolumn{2}{|c|}{ Triacylglycerol } & \multicolumn{2}{|c|}{ LDL-cholesterol } & \multicolumn{2}{|c|}{ SBP } & \multicolumn{2}{|c|}{ AIx } \\
\hline & Coef & $\mathrm{SD}$ & Coef & $\mathrm{SD}$ & Coef & $\mathrm{SD}$ & Coef & $\mathrm{SD}$ \\
\hline Milk & 0.039 & 0.065 & 0.015 & 0.122 & $-10.40 *$ & 4.579 & -0.940 & 1.495 \\
\hline Cheese & -0.025 & 0.030 & $-0.093 *$ & 0.057 & -0.069 & 2.213 & -0.090 & 0.764 \\
\hline Cream & -0.039 & 0.022 & 0.075 & 0.041 & -1.559 & 1.478 & -0.297 & 0.512 \\
\hline Butter & $0.055^{*}$ & 0.032 & $0.141 * *$ & 0.060 & 3.208 & 2.390 & 0.641 & 0.839 \\
\hline DP & $-0.062^{*}$ & 0.032 & -0.027 & 0.061 & -3.645 & 2.401 & $-1.770 *$ & 0.834 \\
\hline $\mathrm{DP}+$ Butter & $-0.093 * *$ & 0.032 & -0.003 & 0.061 & -1.474 & 2.283 & -0.908 & 0.804 \\
\hline
\end{tabular}

Data show coefficient and SD for the highest quartile of intake; LDL; low-density lipoprotein; SBP; systolic blood pressure; DP; milk, cheese and cream; 1, association between average phase 1 to phase 3 dairy intake and outcomes; 2, association between average phase 1 to phase 3 dairy intake and phase 5 outcome; significant trends across quartiles/ groups were at $* P<0.05, * * P<0.01, * * * P<0.001$.

Augmentation index was $1.8 \%$ lower in subjects in the highest quartiles of dairy product intake compared to the lowest $(P$-trend $=0.021)$, whilst in the highest group of milk consumption SBP was $10.4 \mathrm{mmHg}$ lower $(P$-trend $=0.033)$ than the lowest group after a 22.8 -year follow-up - as indicated in the table above. Milk and dairy product intake was not significantly associated with aortic pulse wave velocity. Across increasing quartiles of butter intake, insulin $(P$-trend $=0.011)$, triacylglycerol $(P$-trend $=0.023)$, total cholesterol $(P$-trend $=0.002)$ and LDL-cholesterol concentrations $(P$-trend $=0.010)$ were higher, as was DBP $(P$-trend $=0.027)$. Across increasing groups of milk intake glucose concentrations were lower $(P$-trend $=0.032)$ and across increasing quartiles of dairy products triglyceride concentrations were lower $(P$-trend $=0.031)$. Across increasing quartiles of cheese intake HDL-cholesterol concentrations were higher $(P$-trend $=0.025)$, whilst LDL-cholesterol concentrations were lower $(P$-trend $=0.015)$. The present results confirm that consumption of milk predicts prospective blood pressure, while milk, cheese and cream consumption is not detrimental to arterial stiffness and metabolic markers.

This study was funded by The University of Reading and The Barham Benevolent Trust. The Caerphilly Prospective Study was undertaken by the former MRC Epidemiology Unit and was funded by the Medical Research Council of the United Kingdom. Pulse wave velocity was collected with funding from the BHF.

1. Vlachopoulos C, Aznaouridis K, Stefanadis C (2010) J Am Coll Cardiol 55, 1318-1327.

2. Ralston RA, Lee JH, Truby H et al. (2012) J Hum Hypertens 26, 3-13.

3. Crichton GE, Elias MF, Dore GA et al. (2012) Hypertension 59, 1044-1051. 